\title{
RESEARCH
}

Open Access

\section{Role of ASM/Cer/TXNIP signaling module in the NLRP3 inflammasome activation}

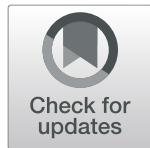

Jianjun Jiang ${ }^{1}$, Yining Shi ${ }^{2}$, Jiyu Cao ${ }^{3}$, Youjin Lu², Gengyun Sun ${ }^{1 *}$ and Jin Yang ${ }^{2^{*}}$

\begin{abstract}
Background: This study aimed to explore the effects of ceramide (Cer) on NLRP3 inflammasome activation and their underlying mechanisms.

Methods: Lipopolysaccharide (LPS)/adenosine triphosphate (ATP)-induced NLRP3 inflammasome activation in J774A.1 cells and THP-1 macrophages was used as an in vitro model of inflammation. Western blotting and realtime PCR (RT-PCR) were used to detect the protein and mRNA levels, respectively. IL-1 $\beta$ and IL-18 levels were measured by ELISA. ASM assay kit and immunofluorescence were used to detect ASM activity and Cer content.

Results: Imipramine, a well-known inhibitor of ASM, significantly inhibited LPS/ATP-induced activity of ASM and the consequent accumulation of Cer. Additionally, imipramine suppressed the LPS/ATP-induced expression of thioredoxin interacting protein (TXNIP), NLRP3, caspase-1, IL-1 $\beta$, and IL-18 at the protein and mRNA level. Interestingly verapamil, a TXNIP inhibitor, suppressed LPS/ATP-induced activation of TXNIP/NLRP3 inflammasome but did not affect LPS/ATP-induced ASM activation and Cer formation. TXNIP siRNA and verapamil inhibited C2-Cerinduced upregulation of TXNIP and activation of the NLRP3 inflammasome. In addition, the pretreatment of cells with sulfo-N-succinimidyl oleate (SSO), an irreversible inhibitor of the scavenger receptor CD36, blocked Cerinduced upregulation of nuclear factor-KB (NF-KB) activity, TXNIP expression, and NLRP3 inflammasome activation. Inhibition of NF-KB activation by SN50 prevented Cer-induced upregulation of TXNIP and activation of the NLRP3 inflammasome but did not affect CD36 expression.
\end{abstract}

Conclusion: This study demonstrated that the ASM/Cer/TXNIP signaling pathway is involved in NLRP3 inflammasome activation. The results documented that the CD36-dependent NF-KB-TXNIP signaling pathway plays an essential role in the Cer-induced activation of NLRP3 inflammasomes in macrophages.

Keywords: Lipopolysaccharide, J774A.1 cells, THP-1 macrophages, Thioredoxin interacting protein, Acid sphingomyelinase, NOD-like receptor Protein3, Ceramide

\footnotetext{
* Correspondence: sungengy@126.com; yangqj1015@foxmail.com

'Department of Respiratory and Critical Care Medicine, the First Affiliated Hospital of Anhui Medical University, 218 Jixi Road, Hefei 230022, Anhui,

China

${ }^{2}$ Department of Respiratory and Critical Care Medicine, the Second Affiliated Hospital of Anhui Medical University, 678 Furong Road, Hefei 230601, Anhui, China

Full list of author information is available at the end of the article
}

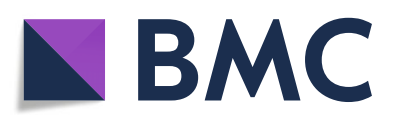

(- The Author(s). 2021 Open Access This article is licensed under a Creative Commons Attribution 4.0 International License, which permits use, sharing, adaptation, distribution and reproduction in any medium or format, as long as you give appropriate credit to the original author(s) and the source, provide a link to the Creative Commons licence, and indicate if changes were made. The images or other third party material in this article are included in the article's Creative Commons licence, unless indicated otherwise in a credit line to the material. If material is not included in the article's Creative Commons licence and your intended use is not permitted by statutory regulation or exceeds the permitted use, you will need to obtain permission directly from the copyright holder. To view a copy of this licence, visit http://creativecommons.org/licenses/by/4.0/. The Creative Commons Public Domain Dedication waiver (http://creativecommons.org/publicdomain/zero/1.0/) applies to the data made available in this article, unless otherwise stated in a credit line to the data. 


\section{Introduction}

Inflammation is a local protective mechanism evoked by the exposure of cells to harmful stimuli, such as irritants, pathogens, or damaged cells. Excessive or chronic inflammation gives rise to multiple pathological complications, such as tissue damage and dysfunction, diabetes, sepsis, arteriosclerosis, Alzheimer's disease, liver disorders, and various malignancies [1]. Commonly prescribed anti-inflammatory drugs fall under two categories: non-steroidal and steroidal. However, these drugs produce a range of side-effects, which restricts their clinical utility $[2,3]$. This limitation necessitates identifying effective alternatives for current antiinflammatory medications.

NOD-Like Receptor Protein 3 (NLRP3) inflammasome is a component of the crucial intracellular inflammatory pathway of the innate immune system. Chronic activation of the NLRP3 inflammasome culminates in the accumulation of pro-inflammatory cytokines, i.e., interleukin-1 $\beta$ (IL-1 $\beta$ ) and interleukin-18 (IL-18), contributing to excessive inflammation and inflammatory disorders [4]. Unraveling the precise regulatory mechanism leading to the activation of NLRP3 inflammasome could help determine molecular drug targets to treat inflammatory diseases.

NLRP3 is activated by exogenous factors, such as pathogens, or endogenous factors, such as ceramide (Cer), an intracellular lipid metabolite $[5,6]$. Recent studies demonstrated that Cer mediates the activation of NLRP3 inflammasome in multiple disorders, such as obesity, glomerular injury, acute lung injury, and Alzheimer's disease [7, 8]. Cer is primarily synthesized through sphingolipid metabolism. Sphingomyelin hydrolysis is one of the first pathways activated during the stress response, and sphingomyelinase serves as a crucial component in the regulation of this pathway [9]. Acid sphingomyelinase (ASM) is localized in the lysosomes and can be activated by diverse stress stimuli, such as oxidative stress, lipopolysaccharide (LPS), and tumor necrosis factor- $\alpha$ (TNF- $\alpha)$. Cer is generated through the ASM-mediated hydrolysis of sphingomyelin at the cell membrane $[10,11]$. Numerous studies have documented that the over-activation of the ASM/Cer pathway constitutes the underlying mechanism for the stimulusinduced inflammation $[12,13]$. We have previously demonstrated that imipramine remarkably reduced the LPSinduced pulmonary inflammation in mice and increased their survival rate by suppressing the intracellular accumulation of Cer [14]. Moreover, NLRP3 shRNA suppressed the Cer-induced activation of NLRP3 inflammasome and secretion of pro-inflammatory cytokines, improving the permeability of type II alveolar epithelial cells [8]. However, further research is necessary on the participation of ASM-hydrolyzed sphingomyelin in the Cer-induced activation of NLRP3 inflammasome in response to stress stimuli. Thioredoxin-interacting protein (TXNIP) can directly interact with and activate the NLRP3 inflammasome [15]. Previous studies have identified the crucial role of the TXNIP/NLRP3 inflammasome signaling pathway in activating the NLRP3 inflammasome. However, whether Cer promotes TXNIP expression in macrophages has not been determined.

CD36 is a multifunctional receptor that binds a variety of ligands [16]. It is expressed in many cell types, including macrophages, adipocytes, endothelial cells, and epithelial cells, and plays an essential role in many different biological processes [16, 17]. In addition, CD36 can act as an independent pattern recognition receptor for various bacteria and bacterial cell wall components and can directly initiate intracellular signaling pathways leading to the release of pro-inflammatory cytokines [18]. Therefore, we investigated whether CD36 mediates the activation of TXNIP and subsequently induces the activation of NLRP3 inflammasomes in macrophages through Cer.

The main objective of this study was to determine whether ASM-derived Cer is involved in the NLRP3 inflammasome activation. We also attempted to identify the mechanism underlying the activation of the NLRP3 inflammasome, with a particular emphasis on the ASM/ Cer/TXNIP signaling pathway.

\section{Materials and methods \\ Materials}

C2-Cer, LPS, adenosine $5^{\prime}$-triphosphate (ATP) disodium salt hydrate, SN50, verapamil, imipramine, sulfo-Nsuccinimidyl oleate (SSO) and phorbol 12-myristate 13acetate (PMA) were acquired from Sigma-Aldrich (St Louis, MO, USA). Anti-NLRP3, anti-TXNIP, anti-CD36, anti-nuclear factor- $\mathrm{B}(\mathrm{NF}-\kappa \mathrm{B})$ and anti-p-NF- $\mathrm{B}$ antibodies were purchased from Abcam (San Francisco, CA, USA). The secondary antibody was obtained from ZSGB-BIO (Beijing, China). Anti-caspase- 1 , anti- $\beta$-actin, and lysis buffer were obtained from Cell Signaling Technology (Beverly, MA, USA).

\section{Cell culture and treatment}

Murine macrophage cell line J774A.1 and human monocyte leukemia cell line (THP-1) were obtained from the University of Science and Technology of China. In the subsequent experiments, THP-1 monocytes were stimulated with PMA $(100 \mathrm{nM})$ for $24 \mathrm{~h}$ to induce them to differentiate into adherent macrophages. The J774A.1 cells and THP-1 macrophages were randomly divided into 1) normal control group, LPS/ATP group, imipramine intervention + LPS/ATP group (cells were incubated with $1 \mu \mathrm{g} / \mathrm{mL}$ LPS for $4 \mathrm{~h}$, treated with $10 \mu \mathrm{mol} / \mathrm{L} \mathrm{im-}$ ipramine for $3 \mathrm{~h}$, and then with $5 \mathrm{mM}$ ATP for $30 \mathrm{~min}$ ), and imipramine control group (imipramine group); 2) 
normal control group, LPS/ATP group, verapamil intervention + LPS/ATP group (cells were incubated with $1 \mu \mathrm{g} / \mathrm{mL}$ LPS for $4 \mathrm{~h}$, treated with $10 \mu \mathrm{mol} / \mathrm{L}$ verapamil for $3 \mathrm{~h}$, and then with $5 \mathrm{mM} \mathrm{ATP}$ for $30 \mathrm{~min}$ ), and verapamil control group (verapamil group); 3) normal control group, Cer group $(30 \mu \mathrm{mol} / \mathrm{L} \mathrm{C} 2-\mathrm{Cer})$, verapamil intervention + Cer group (cells were pretreated with $10 \mu \mathrm{mol} / \mathrm{L}$ verapamil for $3 \mathrm{~h}$ and co-incubated with $30 \mu \mathrm{mol} / \mathrm{L}$ C2-Cer $5 \mathrm{~h}$, and TXNIP siRNA + Cer group (cells were transfected with TXNIP siRNA and incubated with $30 \mu \mathrm{mol} / \mathrm{L} \mathrm{C2}$-Cer for $5 \mathrm{~h}$; and 4) normal control group, Cer group $(30 \mu \mathrm{mol} / \mathrm{L}$ C2-Cer $)$, SSO intervention + Cer group (cells were pretreated with $200 \mu \mathrm{mol} / \mathrm{L} \mathrm{SSO}$ for $1 \mathrm{~h}$ and co-incubated with $30 \mu \mathrm{mol} /$ $\mathrm{L}$ C2-Cer for $5 \mathrm{~h}$ ), SSO control group, SN50 (NF-kB inhibitor) intervention + Cer group (cells were pretreated with $36 \mu \mathrm{mol} / \mathrm{L}$ SN50 for $1 \mathrm{~h}$ and co-incubated with $30 \mu \mathrm{mol} / \mathrm{L} \mathrm{C} 2-\mathrm{Cer}$ for $5 \mathrm{~h}$ ), and SN50 control group.

\section{Transfection}

Control siRNA and TXNIP siRNA were synthesized by GenePharma (Shanghai, China). Cell transfection was performed using Lipofectamine 3000 RNAiMax reagent (Invitrogen, Karlsruhe, Germany) according to the manufacturer's instructions. The cells were collected for Western blotting and RT-PCR at $48 \mathrm{~h}$ post-transfection.

\section{Cell viability assays}

The cells were treated with imipramine $(0,25,50,75$, $100 \mu \mathrm{mol} / \mathrm{L}), \mathrm{C} 2-\operatorname{Cer}(0,15,30,45,60 \mu \mathrm{mol} / \mathrm{L})$ and verapamil $(0,25,50,75,100 \mu \mathrm{mol} / \mathrm{L})$ for $24 \mathrm{~h}$ and then incubated with MTT (Beyotime, Jiangsu, China) for $4 \mathrm{~h}$. Subsequently, dimethylsulfoxide (DMSO) (Beyotime, Jiangsu, China) was added, and the absorbance was measured by the microplate reader (BioTek, Winooski, VT, USA) at $490 \mathrm{~nm}$.

\section{Western blotting}

An equal amount of protein $(10-20 \mu \mathrm{g})$ was taken from each sample, loaded into the individual lane, and subjected to vertical SDS-PAGE electrophoresis (concentration gel voltage $50 \mathrm{~V}, 1 \mathrm{~h}$, and separation gel voltage 100 $\mathrm{V}, 1.5 \mathrm{~h}$ ). The separated proteins were electrophoretically transferred at $200 \mathrm{~mA}$ for $3.5 \mathrm{~h}$ onto a PVDF membrane (Millipore Corporation, Billerica, MA, USA). The membrane was incubated with primary antibodies and corresponding secondary antibodies. Protein bands were scanned using the chemiluminescence imaging system (GE Healthcare, Bucks, UK).

\section{Real-time PCR}

Total RNA was extracted from cells using the Promega reagent (Promega, Beijing, China) according to the manufacturer's instructions. Promega-A3500 kit was used for reverse transcription of RNA and quantitation of cDNA. The $2^{-\Delta \Delta \mathrm{Ct}}$ method was used to calculate the relative expression level of target genes normalized to GAPDH expression.

Primers used in real time-PCR:

\begin{tabular}{lll}
\hline Gene & Forward Primer & Reverse Primer \\
\hline TXNIP & GATACCCCAGAAGCTCCTCC & $\begin{array}{l}\text { ACCTCA GTGTAAGTGG } \\
\text { GTGG }\end{array}$ \\
NLRP3 & AGCCTTCCAGGATCCTCTTC & CTTGGGCAGCAGTTCTTTC \\
Caspase- & TGGCAGGAATCTGGAGCTT & CTTGAGGGTCCCAGTCAG \\
1 & & TC \\
IL-1ß & CTG CTT CCA AAC CTT TGA CC & AGC TTC TCC ACA GCC \\
& & ACA AT \\
IL-18 & AAGACTCTTGCGTCAACT & AGTCGGCCAAAGTGTCT \\
& TCAAGGA & GATTC \\
GAPDH & TGATGGGTGTGACCACGAG & AGTGATGGCATGGACT \\
& & GTGG \\
\hline
\end{tabular}

\section{Elisa}

At the end of each treatment described under section 2.2, the culture medium was collected, and the concentrations of IL-1 $\beta$ and IL-18 were measured using ELISA kits (MultiSciences Biotechnology, Hangzhou, China); manufacturer's instructions were followed. The absorbance of each well was measured on a microplate reader (BioTek) using the wavelength of $450 \mathrm{~nm}$. Each determination was performed in triplicate, the values were averaged, and the cytokine content was calculated using standard curves.

\section{Cer detection by immunofluorescence}

At the end of the treatment (see section 2.2), the cells were fixed, permeabilized, and incubated with the antiCer antibody (1:500, ENZO, Switzerland) at $4{ }^{\circ} \mathrm{C}$ for 12 h. Subsequently, the cells were incubated with the secondary antibody in the dark at room temperature for 1 h. After washing, cells were stained with DAPI in the dark for 10 min. Nikon Eclipse 90i Fluorescence Microscope system (Nikon, Japan) was used to visualize the cells and record the images. The quantification of Cer in THP-1 macrophages utilized the diacylglycerol (DAG) kinase assay, as mentioned earlier [19].

\section{ASM activity measurement}

The cells were lysed using a $1 \mathrm{X}$ mammalian lysis buffer, and the samples were treated with ASM assay reagents (ASM assay kit, Abcam, San Francisco, CA, USA) according to the manufacturer's instructions. After incubating for $1 \mathrm{~h}$ at room temperature, fluorescence was detected using a microplate reader (BioTek) at the excitation and emission wavelength of 540 and $590 \mathrm{~nm}$, respectively. The fluorescence in the blank wells was used as a negative control. 


\section{NF-KB-p65 DNA-binding activity}

The activation of the NF- $\mathrm{kB}$ complex was determined using an ELISA-based p65 transcription factor assay (Cayman Chemicals, Ann Arbor, MI, USA). The nuclear extract was fractionated using the extraction reagent kit (Thermo Fisher Scientific Inc., Rockford, USA) according to the manufacturer's protocol. Oligonucleotides containing p65 uniform binding sites were immobilized in the wells and incubated with nuclear extracts. Finally, after incubation with specific primary and horseradish peroxidase (HRP)-conjugated secondary antibodies, the absorbance was measured at $450 \mathrm{~nm}$.

\section{Statistical analysis}

All experimental data are presented as the mean $\pm \mathrm{SD}$, and statistical analysis was conducted using SPSS version 23.0. The statistical significance of differences between the groups was determined using a one-way analysis of variance (ANOVA). $P<0.05$ was considered to indicated statistical significance.

\section{Results}

Effects of imipramine, C2-Cer, and verapamil on cell viability

The effects of imipramine $(0,25,50,75,100 \mu \mathrm{mol} / \mathrm{L})$, C2-Cer $(0,15,30,45,60 \mu \mathrm{mol} / \mathrm{L})$, and verapamil $(0$, $25,50,75,100 \mu \mathrm{mol} / \mathrm{L})$ on the viability of J774A.1 cells and THP-1 macrophages were measured by the MTT method. Imipramine did not exert significant toxicity in J774A. 1 cells at $0-50 \mu \mathrm{mol} / \mathrm{L}$ and in THP1 macrophages at $0-75 \mu \mathrm{mol} / \mathrm{L}$ (Fig. 1a). C2-Cer at concentrations above $30 \mu \mathrm{mol} / \mathrm{L}$ decreased the viability of both types of cells (Fig. 1b). Verapamil at concentrations up to $50 \mu \mathrm{mol} / \mathrm{L}$ did not affect the viability of cells (Fig. 1c). Base on these results, the concentrations of $10 \mu \mathrm{mol} / \mathrm{L}$ of imipramine, $30 \mu \mathrm{mol} / \mathrm{L}$ of $\mathrm{C} 2$-Cer, and $10 \mu \mathrm{mol} / \mathrm{L}$ of verapamil were used in subsequent experiments.

Imipramine pretreatment inhibits LPS/ATP-induced ASM activity and Cer production

To unravel the role of the ASM/Cer pathway in TXNIP/ NLRP3 inflammasome activation, the effect of imipramine, an ASM activity inhibitor, on LPS/ATPinduced ASM activation and Cer generation was examined in J774A.1 cells and THP-1 macrophages. LPS/ATP significantly increased ASM activity, but this effect was inhibited by imipramine (Fig. 1d). Similarly, LPS/ATP significantly elevated the Cer content, and this effect was blocked by imipramine (Fig. 1e, f, g).
Inhibition of ASM activity attenuates the LPS/ATP-induced TXNIP expression and NLRP3 inflammasome activation in J774A.1 cells and THP-1 macrophages

Next, the effect of imipramine on the LPS/ATPinduced expression of TXNIP protein and the activation of NLRP3 inflammasome was determined. Imipramine suppressed the LPS/ATP-induced expression of TXNIP, NLRP3, and caspase-1 (Fig. 2ae). NLRP3 inflammasome-induced expression of IL-1 $\beta$ and IL-18 was then evaluated to unravel the inhibitory effect of imipramine on NLRP3 inflammasome activation. In this experiment, the upregulation of NLRP3 by LPS/ATP significantly enhanced IL-1 $\beta$ and IL-18 secretion by the cells, and this increase was significantly inhibited by pretreatment with imipramine (Fig. 2f, g).

\section{Inhibition of ASM activity attenuates LPS/ATP-induced NLRP3 inflammasome and TXNIP mRNA expression in J774A. 1 cells and THP-1 macrophages}

The levels of the TXNIP, NLRP3, caspase-1, IL-1 $\beta$, and IL-18 mRNA were significantly increased after treatment with LPS/ATP (Fig. 2h-l). Imipramine significantly inhibited the upregulation of these mRNAs.

\section{Inhibition of TXNIP attenuates LPS/ATP-induced NLRP3 inflammasome activation in J774A.1 cells and THP-1 macrophages}

Effects of verapamil (TXNIP inhibitor) on LPS/ATPinduced TXNIP expression level and NLRP3 inflammasome activation were evaluated next. LPS/ATP upregulated the expression of TXNIP, NLRP3, caspase1 , IL-1 $\beta$, and IL-18, and this effect was mitigated by verapamil (Fig. 3a-g). The impact of verapamil on LPS/ ATP-induced ASM activity and Cer content was also examined. Verapamil did not affect LPS/ATP-induced ASM activity and Cer content (Fig. 1d-f).

Inhibition of TXNIP expression attenuates the Cer-induced NLRP3 inflammasome activation in J774A.1 cells and THP1 macrophages

To determine whether TXNIP/NLRP3 inflammasome is downstream of the ASM/Cer pathway, the effect of verapamil or TXNIP siRNA on C2-Cer induced TXNIP expression and NLRP3 inflammasome activation was examined. siRNA effectively silenced TXNIP target genes (Fig. 3h-j). As expected, C2-Cer upregulated the expression of TXNIP, NLRP3, and caspase-1 protein, and verapamil or TXNIP siRNA suppressed this response (Fig. 3k-o). The effect of verapamil or TXNIP siRNA on C2-Cer-induced secretion of IL-1 $\beta$ and IL-18 was also evaluated. C2-Cer significantly elevated their secretion; however, this effect was 
(A)

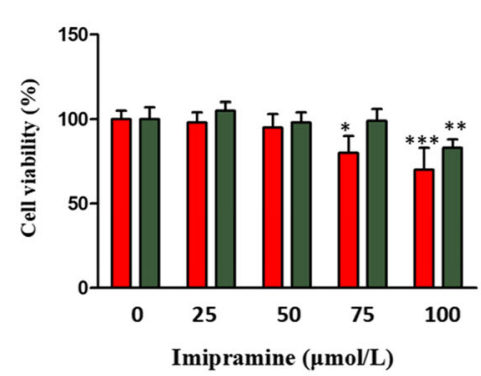

(C)

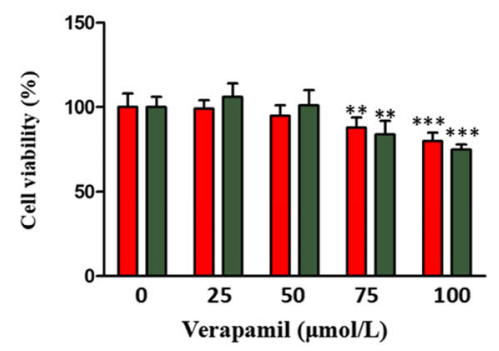

(B)

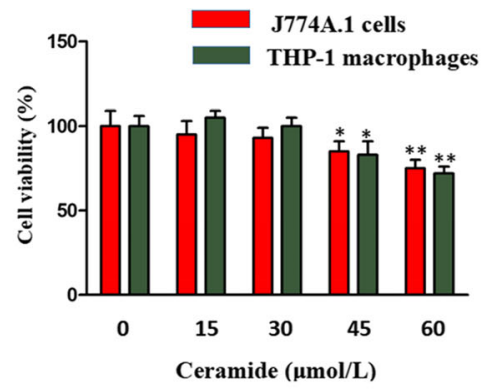

(D)

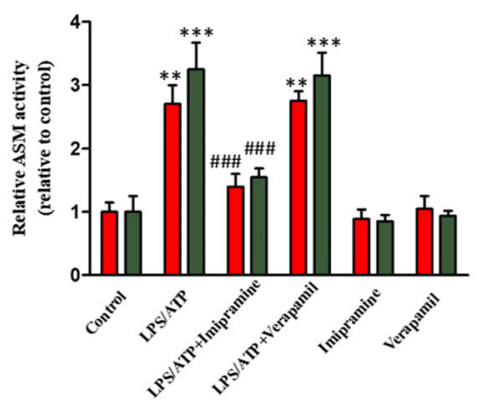

(E)

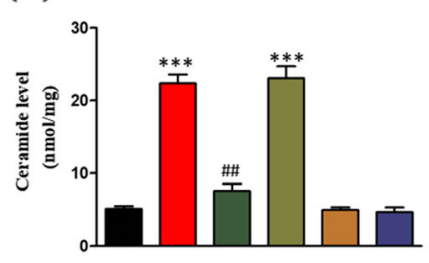

(G)

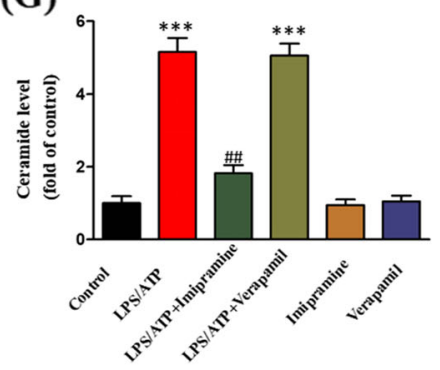

(F)

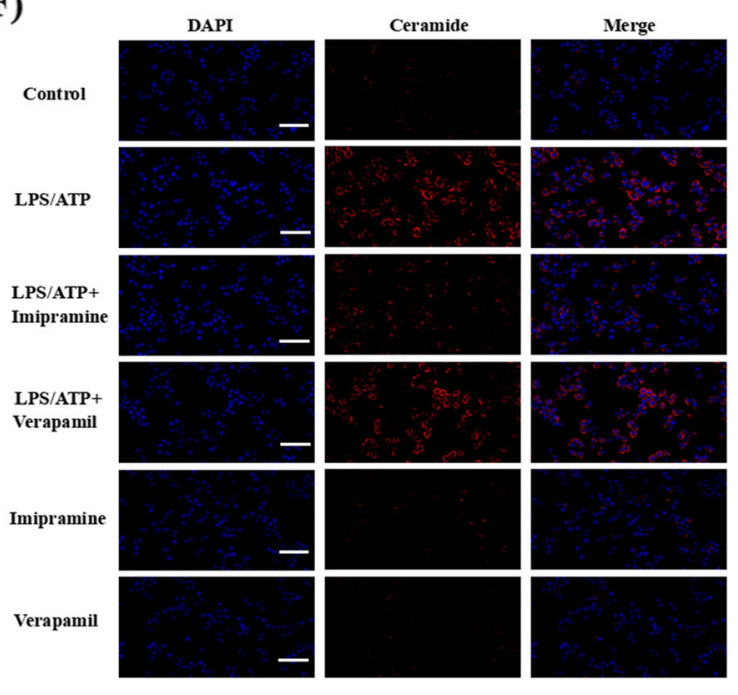

Fig. 1 Effects of imipramine and verapamil on LPS/ATP-induced ASM activity and Cer production. The cells were treated with imipramine $(0,25$, 50, 75, $100 \mu \mathrm{mol} / \mathrm{L}), \mathrm{C} 2-\operatorname{Cer}(0,15,30,45,60 \mu \mathrm{mol} / \mathrm{L})$ and verapamil $(0,25,50,75,100 \mu \mathrm{mol} / \mathrm{L})$ for $24 \mathrm{~h}$. a Imipramine; b C2-Cer; c Verapamil. The J774A.1 cells and THP-1 macrophages were incubated with LPS $(1 \mu \mathrm{g} / \mathrm{mL})$ for $4 \mathrm{~h}$, treated with imipramine or verapamil $(10 \mu \mathrm{mol} / \mathrm{L})$ for $3 \mathrm{~h}$, and finally with ATP $(5 \mathrm{mM})$ for 30 min. d. Effects of imipramine and verapamil on ASM activity in J774A.1 cells and THP-1 macrophages. e Effects of imipramine and verapamil on Cer formation in THP-1 macrophages. $\mathbf{f}, \mathbf{g}$ Effects of imipramine and verapamil on Cer formation in J774A.1 cells. Bar $=50 \mu \mathrm{m} .{ }^{*} P<0.05,{ }^{* *} P<0.01,{ }^{* * *} P<0.001$ vs. control group; ${ }^{\# \#} P<0.01,{ }^{\# \#} P<0.001$ vs. LPS/ATP treatment group

significantly inhibited by pretreatment with verapamil or TXNIP siRNA (Fig. 3p, q).

Inhibition of NF-KB attenuates Cer-induced TXNIP expression and NLRP3 inflammasome activation in J774A.1 cells and THP-1 macrophages

We hypothesized that the Cer-induced up-regulation of TXNIP is related to the activation of NF-kB. NF$\kappa \mathrm{B}$ is a critical nuclear transcription factor regulating inflammation and cell survival. As expected, Cer increased the level of phospho-NF-kB-p65 and increased its binding to DNA in J774A.1 cells and THP-1 macrophages (Fig. 4a-c). However, the NF-kB inhibitor SN5O significantly reduced the NF- $\mathrm{kB}$ DNA binding activity in Cer-treated cells (Fig. 4c). At the same time, SN5O also significantly inhibited the expression of TXNIP and the activation of NLRP3 inflammasome induced by Cer (Fig. 4d-k). 


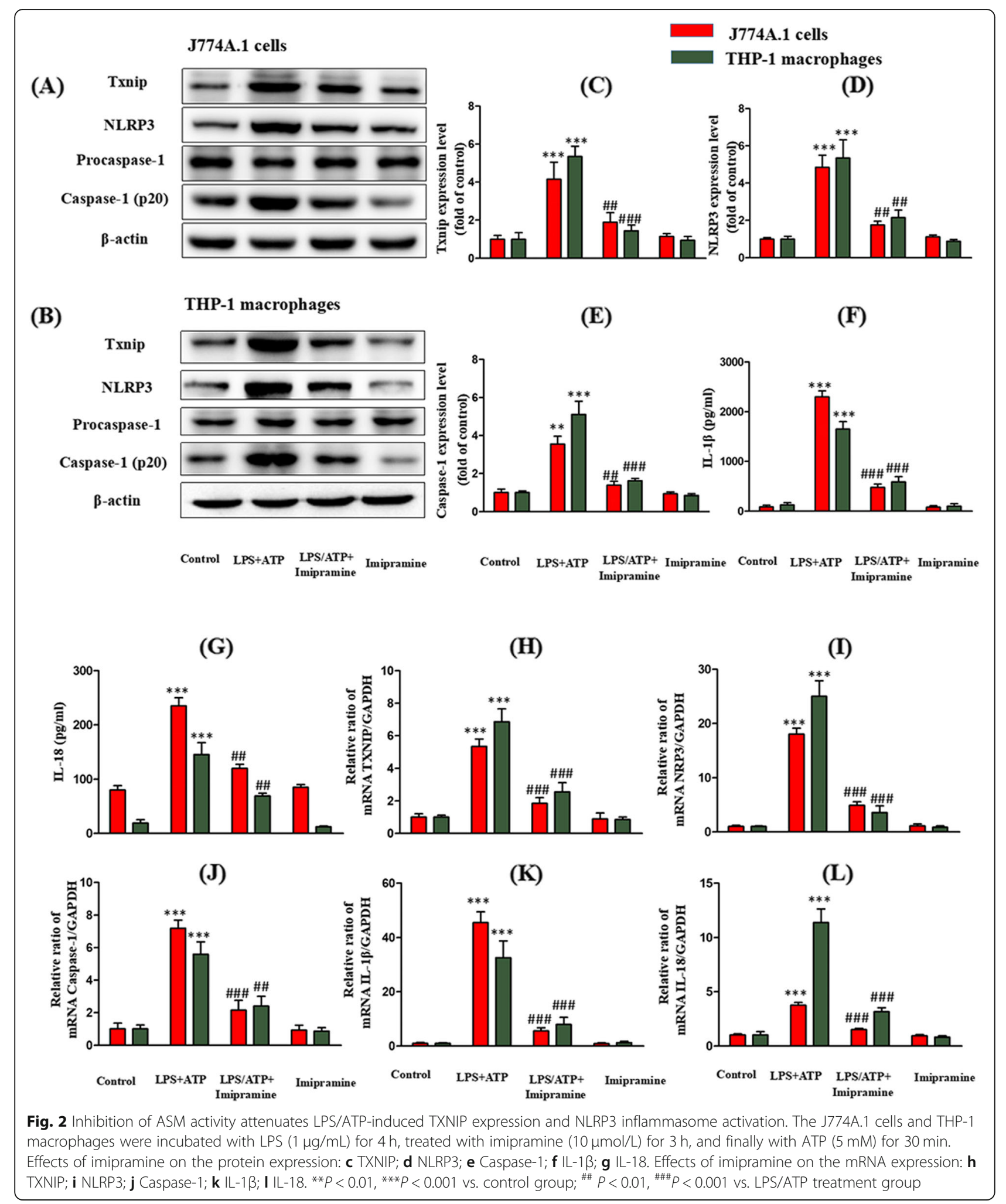




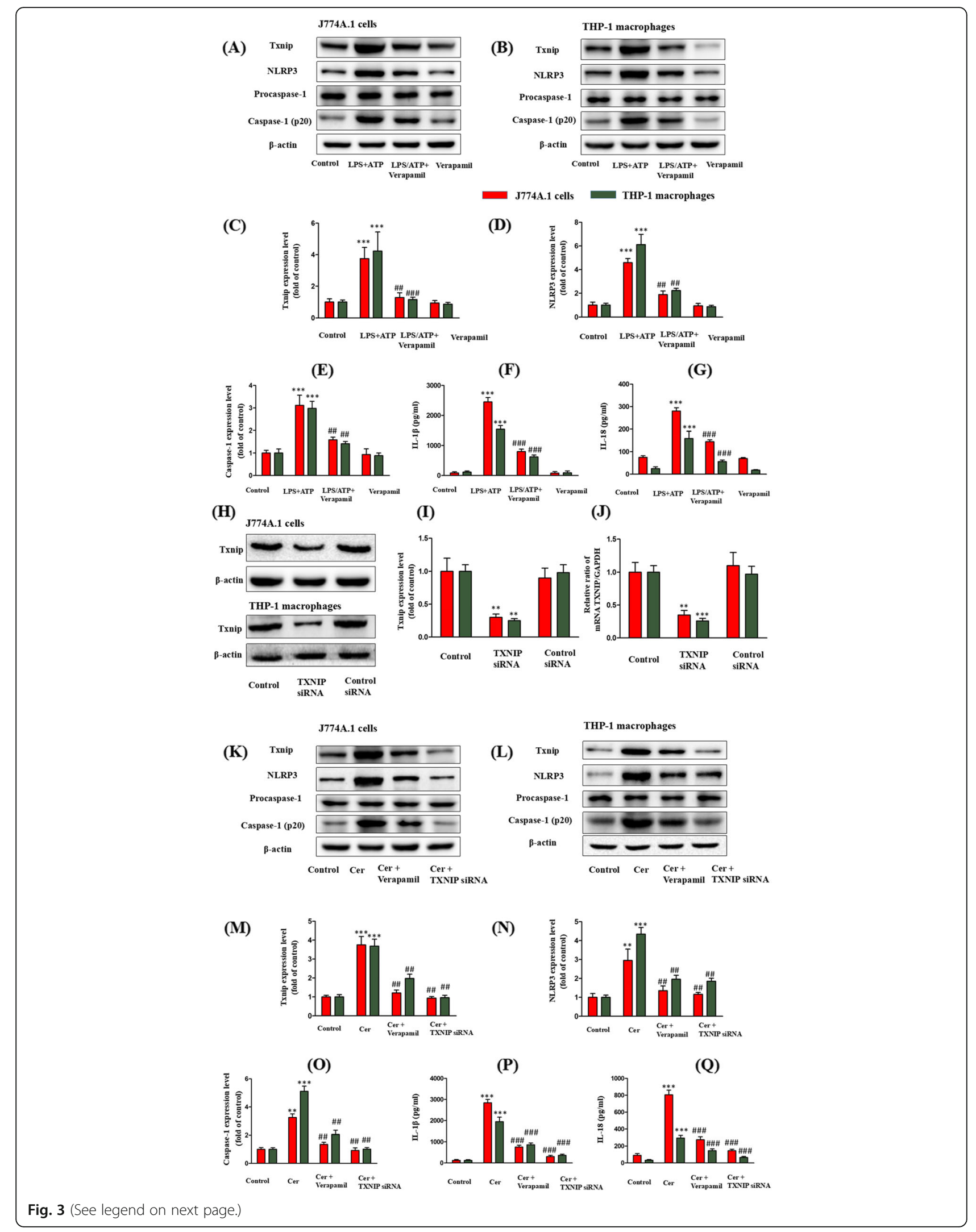


(See figure on previous page.)

Fig. 3 Inhibition of TXNIP attenuates LPS/ATP- or Cer-induced TXNIP expression and NLRP3 inflammasome activation. The J774A.1 cells and THP1 macrophages were incubated with LPS $(1 \mu \mathrm{g} / \mathrm{mL})$ for $4 \mathrm{~h}$, treated with verapamil $(10 \mu \mathrm{mol} / \mathrm{L})$ for $3 \mathrm{~h}$, and finally with ATP $(5 \mathrm{mM})$ for $30 \mathrm{~min}$. Effects of verapamil on the protein expression: $\mathbf{c}$ TXNIP; $\mathbf{d}$ NLRP3; e Caspase-1; $\mathbf{f}\|\mathrm{L}-1 \beta ; \mathbf{g}\| \mathrm{L}-18$. The J774A.1 cells and THP-1 macrophages were pretreated with verapamil $(10 \mu \mathrm{mol} / \mathrm{L})$ for $3 \mathrm{~h}$ or TXNIP siRNA and then incubated with C2-Cer $(30 \mu \mathrm{mol} / \mathrm{L})$ for $5 \mathrm{~h}$. Effects of siRNA on the expression of TXNIP: $\mathbf{i}$ TXNIP protein; $\mathbf{j}$ TXNIP mRNA. Effects of verapamil or TXNIP siRNA on the protein expression: $\mathbf{m}$ TXNIP; $\mathbf{n}$ NLRP3; $\mathbf{o}$ Caspase-

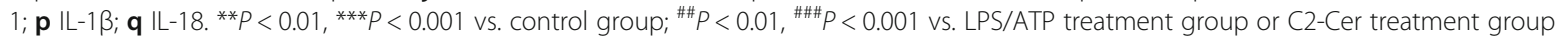

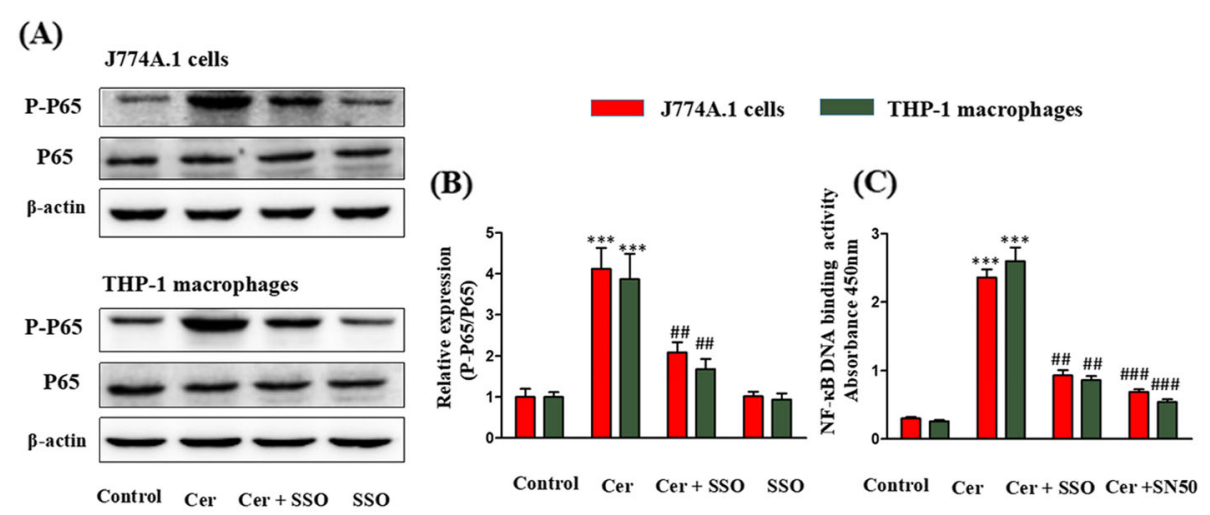

(D)

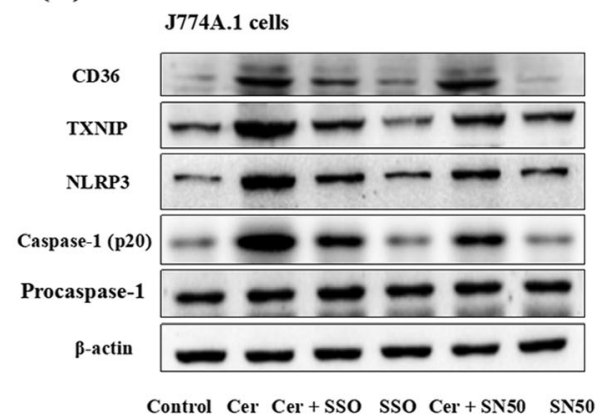

(E)

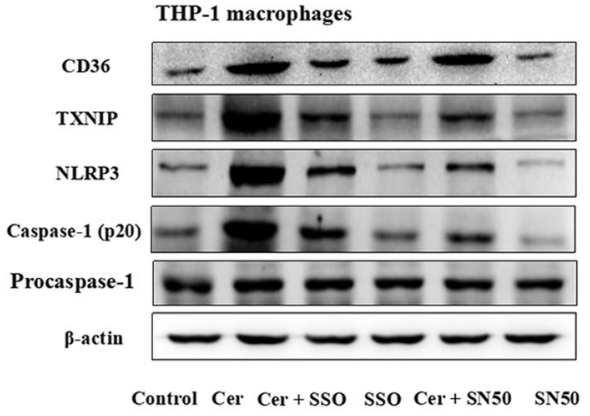

(F)

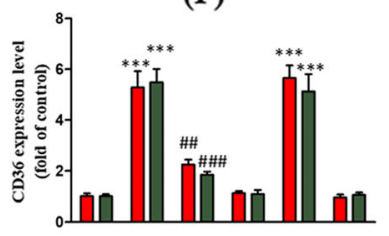

(I)

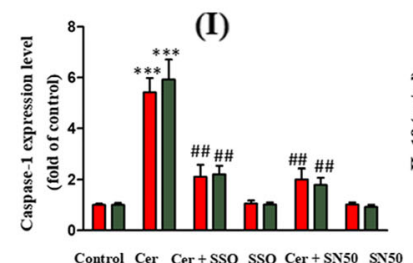

(G)

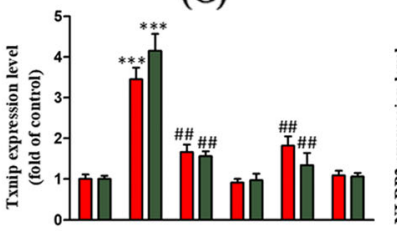

(J)

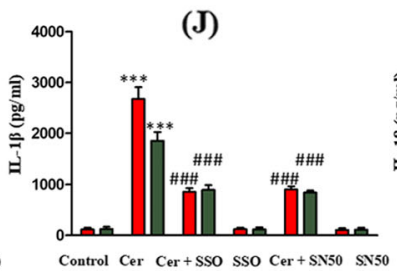

(H)

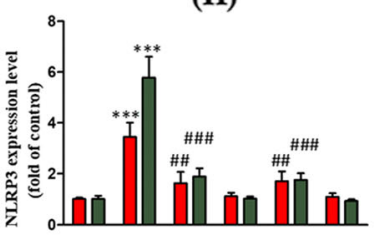

(K)

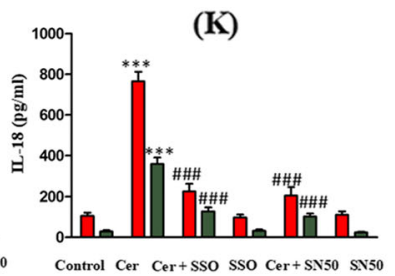

Fig. 4 Inhibition of CD36 attenuates Cer-induced NF-KB activation and prevents subsequent activation of TXNIP/NLRP3 inflammasomes. The

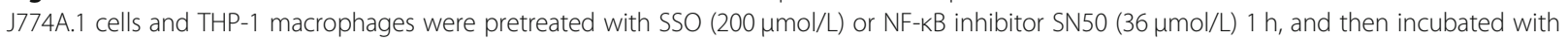
C2-Cer ( $30 \mu \mathrm{mol} / \mathrm{L}$ for $5 \mathrm{~h}$ ). $\mathbf{b}$ Effect of SSO on the activation of phospho-p65. c Effect of SSO and NF-KB inhibitor SN50 on Cer-induced p-P65 DNA-binding activity. Effect of SSO and NF-KB inhibitor SN50 on Cer-induced CD36 expression, TXNIP expression and NLRP3 inflammasomes: $\mathbf{f}$ CD36; $\mathbf{g}$ TXNIP; $\mathbf{h}$ NLRP3; i Caspase-1; $\mathbf{j}\|L-1 \beta ; \mathbf{k}\| L-18$. ${ }^{* *} P<0.01,{ }^{* * *} P<0.001$ vs. control group; ${ }^{\# \#} P<0.01$, ${ }^{\# \# \# ~} P<0.001$ vs. C2-Cer treatment group 
Inhibition of CD36 attenuates Cer-induced NF-KB activation and prevents subsequent activation of TXNIP/ NLRP3 inflammasomes in J774A.1 cells and THP-1 macrophages

The irreversible inhibitor of CD36, sulfo-N-succinimidyl oleate (SSO), inhibited Cer-induced CD36 expression and significantly reduced the increase of phospho-NFкB-p65 and the NF-кB DNA binding activity in Certreated cells (Fig. 4a-f). Therefore, we hypothesized that SSO blocks the Cer-stimulated expression of TXNIP. As expected, pretreatment of cells with SSO significantly reduced the expression of TXNIP and the activation of NLRP3 inflammasome (Fig. 4d-k). Taken together, these results suggest that Cer-mediated TXNIP/NLRP3 activation is regulated by the CD36/NF- $\mathrm{kB}$ pathway.

\section{Discussion}

Macrophages, a vital part of the immune system, play a vital role in the innate immune response [20]. LPS is a crucial component of the extracellular membrane of gram-negative bacteria; it maintains the structural integrity of the cell and elicits pathogen-induced inflammation [21]. The LPS/ATP-stimulated J774A.1 cells and THP-1 macrophages represent a widely accepted in vitro model of inflammation used in the studies on the mechanism of NLRP3 inflammasome activation [22, 23]. Hence, this model was employed in the current study. Accumulating evidence indicates that the aberrant activation or dysregulation of NLRP3 inflammasome is manifested in the most prevalent inflammatory conditions [4]. An in-depth investigation of the signaling pathway implicated in the NLRP3 inflammasome activation could lead to the identification of more relevant drug targets for the effective management of inflammatory disorders. This study demonstrated the involvement of ASM in LPS/ATP-induced Cer production in macrophages, which activated the NLRP3 inflammasome by upregulating TXNIP expression. The obtained results further confirm that the CD36-dependent NF-kBTXNIP signaling pathway plays an important role in the Cer-induced activation of NLRP3 inflammasomes in macrophages.

Sphingolipid metabolism is a significant part of lipid metabolism. It generates an array of active cellular lipids, imparts structural integrity to the cell, and regulates many crucial cellular functions $[24,25]$. A plethora of stress stimuli trigger an excessive formation of Cer through sphingolipid metabolism. As a secondary signaling molecule, Cer activates signaling pathways required to initiate biological processes such as inflammation, apoptosis, and cellular differentiation [26, 27]. Accumulating evidence has demonstrated that Cer accumulation triggers NLRP3 inflammasome activation in various pathological conditions [7, 8]. The intracellular accumulation of Cer occurs through two significant routes of synthesis: de novo synthesis via ceramide synthase serine palmitoyltransferase (SPT) and hydrolysis of the membrane sphingomyelin by neutral sphingomyelinase (NSM)/ASM [26, 28]. However, recent studies suggested that the elevated level of Cer in stress response is an outcome of ASM-mediated sphingomyelin hydrolysis $[9,10]$. Our previous findings have demonstrated that imipramine participates in the amelioration of LPS-induced pulmonary inflammation in mice by suppressing the level of Cer [14]. Imipramine is a wellknown ASM inhibitor, which disrupts the interaction between ASM and lysosomal membrane, eliciting the destruction of ASM by lysosomes [29].

In this study, LPS/ATP treatment significantly elevated the ASM activity and the Cer level, and these changes were significantly attenuated by the ASM inhibitor imipramine. This finding implies the presence of a positive correlation between the LPS/ATP-induced Cer accumulation and ASM activation in J774A.1 cells and THP-1 macrophages. Additionally, imipramine suppressed LPS/ATP-induced activation of TXNIP/NLRP3 inflammasome. TXNIP contributes significantly to biological processes, such as inflammation, oxidative stress, cell apoptosis, and glucose and lipid metabolism [30]. These processes, which are major contributors to the onset of inflammatory disorders, are negatively impacted by the elevated level of TXNIP $[31,32]$. It was previously shown that TXNIP serves the crucial task of NLRP3 assembly by directly interacting with NLRP3 [15, 32]. However, its role in ASM/Cer-mediated TXNIP/NLRP3 inflammasome activation remains unexplored, prompting us to investigate the involvement of the ASM/Cer signaling pathway as an upstream regulator of TXNIP/ NLRP3 inflammasome activation. The TXNIP inhibitor verapamil has been widely employed in the management of inflammation to suppress TXNIP expression and the associated NLRP3 inflammasome activation [33]. In the current study, verapamil suppressed the LPS/ATP-induced TXNIP/NLRP3 inflammasome activation. However, verapamil did not affect LPS/ATP-induced activation of ASM and production of Cer. Therefore, J774A.1 cells and THP-1 macrophages were stimulated with C2-Cer to establish whether the TXNIP/NLRP3 inflammasome activation is a downstream event of the ASM/Cer signaling pathway. This experiment showed that $\mathrm{C} 2$-Cer significantly increased the expression of TXNIP, NLRP3, and caspase-1 proteins, and the secretion of IL-1 $\beta$ and IL-18. Furthermore, verapamil or TXNIP siRNA inhibited C2-Cer-mediated upregulation of TXNIP and activation of the NLRP3 inflammasome, suggesting that Cer induces TXNIP overexpression and subsequent NLRP3 inflammasome activation. Given that a high expression of TXNIP is associated with multiple 
pathological processes, further research is needed to identify the molecules that affect the regulation of TXNIP expression by Cer. The recognition of these molecules may lead to the identification of new drug targets. In this context, it is interesting that the Cer-induced activation of NF- $\mathrm{KB}$ is accompanied by an increase in TXNIP expression [34].

Recent results indicate that the NF- $\mathrm{kB}$ pathway is also involved in the regulation of NLRP3 inflammasome activation [35]. Cer is an effective inducer of the inflammatory response by activating NF-kB $[35,36]$. However, the pathogenic role of the relationship among the NF- $\mathrm{KB}$ signaling pathway, TXNIP, and NLRP3 during Cer-induced NLRP3 inflammasome activation has not been fully understood. Of relevance, the NF- $\kappa B$ inhibitor SN-50 significantly inhibits Cer-induced NF- $\mathrm{kB}$ activation, TXNIP expression, and NLRP3 inflammasome activation. Therefore, it can be concluded that the activation of NF- $\mathrm{kB}$ is a key factor in the induction of TXNIP expression and NLRP3 inflammasome activation by Cer in macrophages. Previous studies have shown that Cer increases the expression of CD36 and that CD36 has an essential function in the activation of NLRP3 inflammasomes in macrophages [34, 37]. The current work demonstrated that Cer increases the expression of CD36 in macrophages, and the inhibition of CD36 by SSO significantly reduced Cer-induced TXNIP expression and NLRP3 inflammasome activation. Interestingly, the inhibitory effect of SSO on CD36 also significantly reduced the Cer-induced activation of NF- $\mathrm{kB}$, which is upstream of TXNIP, but the inhibition of NF- $\mathrm{KB}$ activation did not affect the expression of CD36. In agreement with our findings, the activation level of NF-kB was significantly reduced in macrophages from CD36-deficient patients and CD36 knock-out mice $[38,39]$. In addition, the activation of NF- $\mathrm{kB}$ and the release of IL- $1 \beta$ and TNF- $\alpha$ in CD36-deficient macrophages were significantly reduced [40]. In summary, these results reveal the critical role of the activation of the CD36-NF- $\mathrm{KB}$ signaling pathway in Cer-induced TXNIP expression and NLRP3 inflammasome activation.

\section{Study strengths and limitations}

The advantages of this study are as follows. Firstly, the experiments documented that LPS/ATP induces Cer accumulation in macrophages by activating ASM, leading to subsequent activation of NLRP3 inflammasomes. Secondly, the study identified the involvement of the ASM/Cer/TXNIP signaling pathway in NLRP3 inflammasome activation. Thirdly, the CD36dependent NF- $\mathrm{kB} / \mathrm{TXNIP}$ signaling pathway was found to be essential for the Cer-induced activation of NLRP3 inflammasomes in macrophages. However, this study also has certain limitations. The results were derived from cell experiments in vitro, necessitating their verification by animal experiments in vivo.

\section{Conclusions}

In conclusion, this study demonstrated the crucial role of the ASM/Cer/TXNIP signaling pathway in NLRP3 inflammasome activation. The important function of the CD36-NF- $\mathrm{BB}$ signaling pathway in the Cer-induced upregulation of TXNIP and subsequent activation of NLRP3 inflammasome was also shown. Further understanding of the mechanism of NLRP3 inflammasome activation might reveal novel approaches to the treatment of inflammatory diseases, such as acute lung injury and glomerular injury. The targeted regulation of the Cer signaling pathway may provide innovative therapeutic strategies for inflammatory diseases.

\section{Abbreviations \\ ASM: Acid sphingomyelinase; ATP: Adenosine triphosphate; Cer: Ceramide; ELISA: Enzyme-linked immunosorbent assay; DAG: Diacylglycerol; \\ DMSO: Dimethylsulfoxide; HRP: Horseradish peroxidase; IL-18: Interleukin-18; IL-1 B: Interleukin-1 B; LPS: Lipopolysaccharide; NF-KB: Nuclear factor-kB; NLRP3: NOD-like receptor protein 3; NSM: Neutral sphingomyelinase; PMA: Phorbol 12-myristate 13-acetate; RT-PCR: Real-time PCR; SPT: Serine palmitoyltransferase; SSO: Sulfo-N-succinimidyl oleate; TXNIP: Thioredoxin interacting protein; TNF-a: Tumor necrosis factor-a}

\section{Acknowledgments \\ None.}

\section{Authors' contributions}

Jianjun Jiang wrote this paper. Jianjun Jiang and Yining Shi conducted the experiments. Jin Yang and Youjin Lu analyzed the data. Jiyu Cao and Gengyun Sun supervised the study. The author(s) read and approved the final manuscript.

\section{Funding}

This study was supported by a grant from the National Natural Science Foundation of China (grant number 81400058).

Availability of data and materials

Data are available from the authors upon request.

Ethics approval and consent to participate

Not applicable.

Consent for publication

Not applicable.

Competing interests

The authors declare that they have no competing interests.

\section{Author details}

'Department of Respiratory and Critical Care Medicine, the First Affiliated Hospital of Anhui Medical University, 218 Jixi Road, Hefei 230022, Anhui, China. ${ }^{2}$ Department of Respiratory and Critical Care Medicine, the Second Affiliated Hospital of Anhui Medical University, 678 Furong Road, Hefei 230601, Anhui, China. ${ }^{3}$ Department of Occupational Health and

Environmental Health, School of Public Health, Anhui Medical University, 81 Meishan Road, Hefei 230032, Anhui, China.

Received: 30 August 2020 Accepted: 8 February 2021

Published online: 21 February 2021

\footnotetext{
References

1. Nathan C. Points of control in inflammation. Nature. 2002;420:846-52.

2. Elsaman T, Aldeeb OAA, Aboul-Fadl T, Hamedelneil El. Synthesis, characterization and pharmacological evaluation of certain enzymatically cleavable NSAIDs amide prodrugs. Bioorg Chem. 2017;70:144-52.
} 
3. Uchiyama E, Papaliodis GN, Lobo A-M, Sobrin L. Side-effects of antiinflammatory therapy in uveitis. Semin Ophthalmo. 2014;29:456-67.

4. Elliott El, Sutterwala FS. Initiation and perpetuation of NLRP3 inflammasome activation and assembly. Immunol Rev. 2015;265:35-52.

5. Liston A, Masters SL. Homeostasis-altering molecular processes as mechanisms of inflammasome activation. Nat Rev Immunol. 2017:17:208-14.

6. Camell CD, Nguyen KY, Jurczak MJ, Christian BE, Shulman Gl, Shadel GS, et al. Macrophage-specific de novo synthesis of Ceramide is dispensable for Inflammasome-driven inflammation and insulin resistance in obesity. J Biol Chem. 2015;290:29402-13.

7. Li C, Guo S, Pang W, Zhao Z. Crosstalk between acid Sphingomyelinase and Inflammasome signaling and their emerging roles in tissue injury and fibrosis. Front Cell Dev Biol. 2020;7:378.

8. Lauterbach MA, Saavedra V, Mangan MSJ, Penno A, Thiele C, Latz E, et al. 1-Deoxysphingolipids cause autophagosome and lysosome accumulation and trigger NLRP3 inflammasome activation [published online ahead of print, 2020 Aug 24]. Autophagy. 2020;1-15. https://doi.org/10.1080/1554 8627.2020.1804677.

9. Insausti-Urkia N, Solsona-Vilarrasa E, Garcia-Ruiz C, Fernandez-Checa JC. Sphingomyelinases and liver diseases. Biomolecules. 2020;10:1497.

10. Pandolfi R, Barreira B, Moreno E, Lara-Acedo V, Morales-Cano D, MartinezRamas A, et al. Role of acid sphingomyelinase and IL-6 as mediators of endotoxin-induced pulmonary vascular dysfunction. Thorax. 2017;72:460-71.

11. Wang J, Pendurthi UR, Rao LVM. Acid sphingomyelinase plays a critical role in LPS- and cytokine-induced tissue factor procoagulant activity. Blood. 2019;134:645-55.

12. Cheng Y-P, Zhang H-J, Su Y-T, Meng X-X, Xie X-P, Chang Y-M, et al. Acid sphingomyelinase/ceramide regulates carotid intima-media thickness in simulated weightless rats. Pflugers Arch. 2017;469:751-65.

13. Meng X, Gu Z, Xie X, Su Y, Zhang X, Ma H, et al. Acid sphingomyelinase mediates the noise-induced liver disorder in mice. Clin Exp Pharmacol Physiol. 2019;46:556-66.

14. Yang J, Qu J-m, Summah H, Zhang J, Zhu Y-g, Jiang H-n. Protective effects of imipramine in murine endotoxin-induced acute lung injury. Eur $\rfloor$ Pharmacol. 2010;638:128-33.

15. Zhou R, Tardivel A, Thorens B, Choi I, Tschopp J. Thioredoxin-interacting protein links oxidative stress to inflammasome activation. Nat Immunol. 2010;11:136-51.

16. Yang M, Silverstein RL. CD36 signaling in vascular redox stress. Free Radic Biol Med. 2019;136:159-71.

17. Silverstein RL, Febbraio M. CD36, a scavenger receptor involved in immunity, metabolism, angiogenesis, and behavior. Sci Signal. 2009;2:re3.

18. Baranova IN, Kurlander R, Bocharov AV, Vishnyakova TG, Chen Z, Remaley AT, et al. Role of human CD36 in bacterial recognition, phagocytosis, and pathogen-induced JNK-mediated signaling. J Immunol. 2008;181:7147-56.

19. Zhao M, Pan W, Shi R-z, Bai Y-p, You B-y, Zhang K, et al. Acid sphingomyelinase mediates oxidized-LDL induced apoptosis in macrophage via endoplasmic reticulum stress. J Atheroscler Thromb. 2016;23:1111-25.

20. Koenderman L, Buurman W, Daha MR. The innate immune response. Immunol Lett. 2014;162:95-102.

21. Piazza M, Calabrese V, Baruffa C, Gioannini T, Weiss J, Peri F. The cationic amphiphile 3,4-bis (tetradecyloxy) benzylamine inhibits LPS signaling by competing with endotoxin for CD14 binding. Biochem Pharmacol. 2010;80: 2050-6

22. Hung Y-L, Wang S-C, Suzuki K, Fang S-H, Chen C-S, Cheng W-C, et al. Bavachin attenuates LPS-induced inflammatory response and inhibits the activation of NLRP3 inflammasome in macrophages. Phytomedicine. 2019;59:152758.

23. Zhang $Y$, Jiang $M$, Cui B-W, Jin CH, Wu Y-L, Shang $Y$, et al. P2X7 receptortargeted regulation by tetrahydroxystilbene glucoside in alcoholic hepatosteatosis: a new strategy towards macrophage-hepatocyte crosstalk. Br J Pharmacol. 2020;177:2793-811.

24. Borodzicz S, Czarzasta K, Kuch M, Cudnoch-Jedrzejewska A. Sphingolipids in cardiovascular diseases and metabolic disorders. Lipids Health Dis. 2015;14:55

25. Agudelo CW, Samaha G, Garcia-Arcos I. Alveolar lipids in pulmonary disease A review. Lipids Health Dis. 2020;19:122.

26. Petrache I, Natarajan V, Zhen $L$, Medler TR, Richter AT, Cho C, et al. Ceramide upregulation causes pulmonary cell apoptosis and emphysemalike disease in mice. Nat Med. 2005;11:491-8.

27. Song Y, Yu Q, Zhang J, Huang W, Liu Y, Pei H, et al. Increased myocardial ischemia-reperfusion injury in renal failure involves cardiac adiponectin signal deficiency. Am J Physiol Endocrinol Metab. 2014;306:E1055-64.
28. Goggel R, Winoto-Morbach S, Vielhaber G, Imai Y, Lindner K, Brade L, et al. PAF-mediated pulmonary edema: a new role for acid sphingomyelinase and ceramide. Nat Med. 2004;10:155-60.

29. Hurwitz R, Ferlinz K, Sandhoff K. The tricyclic antidepressant desipramine causes proteolytic degradation of lysosomal sphingomyelinase in human fibroblasts. Biol Chem Hoppe Seyler. 1994;375:447-50.

30. Yoshihara E, Masaki S, Matsu Y, Chen Z, Tian H, Yodoi J. Thioredoxin/Txnip: redoxisome, as a redox switch for the pathogenesis of diseases. Front Immunol. 2014;4:514

31. Perrone L, Devi TS, Hosoya Kl, Terasaki T, Singh LP. Inhibition of TXNIP expression in vivo blocks early pathologies of diabetic retinopathy. Cell Death Dis. 2010;1:e65.

32. Shah A, Xia L, Masson EAY, Gui C, Monnen A, Shikatani EA, et al. Thioredoxin-interacting protein deficiency protects against diabetic nephropathy. J Am Soc Nephrol. 2015:26:2963-77.

33. Chen J, Cha-Molstad H, Szabo A, Shalev A. Diabetes induces and calcium channel blockers prevent cardiac expression of proapoptotic thioredoxininteracting protein. Am J Physiol Endocrinol Metab. 2009;296:E1133-9.

34. Karunakaran U, Elumalai S, Moon JS, Won KC. CD36 dependent redoxosomes promotes ceramide-mediated pancreatic beta-cell failure via p66Shc activation. Free Radic Biol Med. 2019;134:505-15.

35. Boaru SG, Borkham-Kamphorst E, Van de Leur E, Lehnen E, Liedtke C, Weiskirchen R. NLRP3 inflammasome expression is driven by NF-kappa B in cultured hepatocytes. Biochem Biophys Res Commun. 2015;458:700-6.

36. Liang X, Zhang D, Liu W, Yan Y, Zhou F, Wu W, et al. Reactive oxygen species trigger NF-KB-mediated NLRP3 inflammasome activation induced by zinc oxide nanoparticles in A549 cells. Toxicol Ind Health. 2017;33:737-45.

37. Sheedy FJ, Grebe A, Rayner KJ, Kalantari P, Ramkhelawon B, Carpenter SB, et al. CD36 coordinates NLRP3 inflammasome activation by facilitating intracellular nucleation of soluble ligands into particulate ligands in sterile inflammation. Nat Immunol. 2013:14:812-20.

38. Janabi M, Yamashita S, Hirano K, Sakai N, Hiraoka H, Matsumoto K, et al. Oxidized LDL-induced NF-kappa B activation and subsequent expression of proinflammatory genes are defective in monocyte-derived macrophages from CD36-deficient patients. Arterioscler Thromb Vasc Biol. 2000;20:1953-60.

39. Kunz A, Abe T, Hochrainer K, Shimamura M, Anrather J, Racchumi G, et al. Nuclear factor-kappa B activation and postischemic inflammation are suppressed in CD36-null mice after middle cerebral artery occlusion. J Neurosci. 2008;28:1649-58.

40. Yamashita S, Hirano K-i, Kuwasako T, Janabi M, Toyama Y, Ishigami M, et al. Physiological and pathological roles of a multi-ligand receptor CD36 in atherogenesis; insights from CD36-deficient patients. Mol Cell Biochem. 2007;299:19-22.

\section{Publisher's Note}

Springer Nature remains neutral with regard to jurisdictional claims in published maps and institutional affiliations.
Ready to submit your research? Choose BMC and benefit from:

- fast, convenient online submission

- thorough peer review by experienced researchers in your field

- rapid publication on acceptance

- support for research data, including large and complex data types

- gold Open Access which fosters wider collaboration and increased citations

- maximum visibility for your research: over $100 \mathrm{M}$ website views per year

At $\mathrm{BMC}$, research is always in progress.

Learn more biomedcentral.com/submissions 